\title{
Pressing Intervention Promotes the Skeletal Muscle Repair of Traumatic Myofascial Trigger Points in Rats
}

\author{
Quanrui Jiang \\ Xiang Feng (D) \\ Dan Liu \\ Tao Li \\ Xiaoxia Kuang \\ Xiaowei Liu \\ $\mathrm{Wu} \mathrm{Li}$ \\ Jiangshan Li
}

College of Acupuncture \& Moxibustion and Tui-Na, Hunan University of Chinese Medicine, Changsha, 4I0208, People's Republic of China
Correspondence: Jiangshan Li; Wu Li Tel +86-I37073 I5642; +86- |357485 I647 Email 292463008@qq.com;

272434694@qq.com
Objective: To observe the effect of pressing intervention on the skeletal muscle repair of myofascial trigger points (MTrPs) in rats and explore the mechanism of pressing intervention on the deactivation of trigger points.

Methods: Thirty SPF rats were randomly divided into blank group, model group and press group, with 10 rats in each group. The MTrPs models were established by blunt striking plus eccentric exercise, and then evaluated. The press group was given a pressing intervention with a self-made device for 14 days, and the rats in the other two groups were fed normally. Soft tissue tension (STT) $\mathrm{D}_{0.2}$ and pressure pain threshold (PPT) were measured before and after intervention. The skeletal muscle tissue at MTrPs was extracted and assessed by hematoxylin-eosin (HE) and Masson staining. The expression of collagen I, collagen III, $\alpha$ - smooth muscle actin ( $\alpha$-SMA), myosin heavy chain (MHC) and fibronectin (FN) were detected by Western Blotting. Enzyme linked immunosorbent assay (ELISA) was used to evaluate the expression of substance P (SP), 5-hydroxytryptamine (5-HT), cyclooxygenase 2 (COX-2) and prostaglandin E2 (PGE2).

Results: (1) Compared with the blank group, the PPT and $\mathrm{D}_{0.2}$ reduced $(\mathrm{P}<0.05)$ in the model group; while compared with the model group, the PPT and $\mathrm{D}_{0.2}$ increased $(\mathrm{P}<0.05)$ in the press group. (2) Compared with the blank group, the model group showed obvious spontaneous potentials with higher amplitude and frequency, which were also much higher than those of the press group $(\mathrm{P}<0.05)$. (3) The HE and Masson staining results showed evident fibrosis in the muscle tissue of the model group, with a larger area of collagen fibers relative to that of the press group $(\mathrm{P}<0.05)$. (4) The amount of collagen I, collagen III, FN, $\alpha$ - SMA, SP, 5-HT, COX-2 and PGE2 increased and the content of MHC decreased $(\mathrm{P}<$ $0.05)$ in the model group, as compared to the blank group; while all the substances $(\mathrm{P}<$ $0.05)$, instead of MHC which increased $(\mathrm{P}<0.05)$, in the press group were decreased as compared to the model group.

Conclusion: Pressing intervention on the MTrPs in rats can alleviate chronic inflammation, inhibit fibrosis, promote skeletal muscle repair and relieve pain.

Keywords: pressing, myofascial trigger points, skeletal muscle, chronic inflammation, fibrosis

\section{Introduction}

Myofascial trigger points (MTrPs) are hyperirritable spots in skeletal muscle and correlate to small hypersensitive nodules within taut bands on palpation. Clinically, they are commonly associated with symptoms including tenderness, referred pain, motor dysfunction and autonomic symptoms, ${ }^{1}$ which are generally manifestations 
of myofascial pain syndrome (MPS). Due to the relatively subjective diagnosis and lack of consensus, MPS is easy to be confused with other diseases. In 2019, the International Classification of Diseases 11 (ICD-11) formally introduced the concept of chronic primary/secondary skeletal muscle pain. ${ }^{2}$ According to reported surveys, MTrPs present a high detection rate in many common pain conditions and are associated with a variety of diseases. ${ }^{3,4}$ Beside, the related symptoms are readily recurrent and some of them, such as pain, depressive states and insomnia, would cause a serious decrease in the quality of learning, life and work. ${ }^{5}$

According to the integrated hypothesis, the occurrence of MTrPs is associated with a vicious cycle involving abnormal motor endplate (neuromuscular junction), acetylcholine accumulation, impaired local circulation and energy metabolism, as well as abnormal release of vasoactive components and inflammatory factors. ${ }^{6}$ Local trauma and eccentric exercise are important predisposing factors. ${ }^{7,8}$ During the repair of long-term damaged skeletal muscle, the development of chronic inflammation would deposit massive extracellular matrix (ECM) substances such as collagen I, collagen III and fibronectin (FN), leading to skeletal fibrosis, which would reduce skeletal muscle function, biomechanical properties and exercise capacity. ${ }^{9}$ In the meantime, production of cyclooxygenase 2 (COX-2) and prostaglandin E2 (PGE2) by monocytes and macrophages would be largely increased, promoting the recruitment of substance P (SP) derived from fine fiber endings into the body fluid to stimulate mast cells and platelets, followed by release of histamine and 5-hydroxytryptamine (5-HT) contributing to activation of adjacent injurious receptors. All the substances together participate in the generation of inflammatory pain in the body. ${ }^{10}$

Clinically, MTrPs are generally managed by physiotherapy interventions such as acupuncture, massage and cupping, which could deactivate MTrPs thus to relieve symptoms. ${ }^{11}$ Pressing is a type of manual therapy belonging to the massage category, which is noninvasive, effective, cost-effective and has a safety profile. It can provide persistent vertical pressure on MTrPs or specific regions using hands or aids, which allows the taut band to relax. ${ }^{12}$ There were multiple clinical studies reporting that pressing on MTrPs could alleviate muscular pain, contributing to increased function and range of movement. ${ }^{13-15}$ Moreover, pressing could be applied in combination with other therapies to bring more favorable efficacy, for example, alleviate the sore feeling resulted from acupuncture on
MTrPs. ${ }^{16,17}$ Despite the definite clinical efficacy of pressing treatment, its mechanism has not been clarified yet. In our previous animal studies, we found that the muscle fibers of the MTrPs in rats were broken accompanied by a disordered A-belt, the blurred or even disappeared M-line, and the twisted Z-line. With pressing intervention, the muscle fibers tended to be much more organized and integrated. ${ }^{18}$ As regards the underlying mechanism, we hypothesize that pressing could alleviate chronic inflammation and inhibit fibrosis, consequently promoting skeletal muscle repair and relieving pain.

\section{Materials and Methods}

\section{Experimental Animals}

Thirty male SD rats (SPF) weighing 250 280 g were provided by Hunan Slack Jingda Experimental Animal Co., Ltd (Animal license: SYXK 2019-0009). All animals were housed in separate cages in the Experimental Animal Center of Hunan University of Chinese Medicine, with three animals per cage under an agreeable sterilized living conditions $\left(24-26^{\circ} \mathrm{C}\right.$; humidity: $50-70 \% ; 12 / 12$ light/ dark). Food and water were provided ad libitum. All rats were randomly divided into blank, MTrPs model and press groups, with 10 rats in each group. The experimental procedure was approved by the experimental animal ethics committee of Hunan University of Chinese Medicine. The experimental process strictly complies with the Guidance Opinions on Treating Experimental Animals well issued by the Ministry of Science and Technology in 2006.

\section{Main Reagents and Instruments}

Respiratory anesthesia machine (Ruiward Life Technology Co., Ltd, R500), Treadmill set (Zhejiang Jinhua Yusheng Sports Co., Ltd, C100), Therapeutic pressing instrument and blunt striker (self-made), Mechanical pressure pain threshold meter (Tianjin Mingtong Century Technology Co., Ltd, YT-10C), Soft tissue tension meter (Tianjin Mingtong Century Technology Co., Ltd, JZL-III), Electromyography (BIOPAC, MP150), Electrophoresis system (Bio-Rad), Trans-Blot Turbo Transfer System (Bio-Rad), Western developer (Tanon5200), Slicer (Thermo Fisher, MH325), Embedding machine (KEDEE, KD-BM II), Optical microscope (Motic, BA410E), Isoflurane (Ruiward Life Technology Co., Ltd, R510-22), 4\% paraformaldehyde (Biosharp), Anti-collagen I antibody (Proteintech, 14695-1-AP), Anti-collagen III antibody (Hunan AiFang Biology, AF10773), Anti- $\alpha$-smooth 
muscle actin ( $\alpha$-SMA) antibody (Hunan AiFang Biology, AF10987), Anti-myosin heavy chain (MHC) antibody (Proteintech, 10799-1-AP), Anti-Fibronectin antibody (Hunan AiFang Biology, AF10787), Anti-GAPDH antibody (Immunoway, YM3029), HRP-conjugated goat antimouse IgG (Proteintech, SA00001-1), HRP-conjugated goat anti-rabbit IgG (Proteintech, SA00001-2), Rat SP ELISA Kit (Jiangsu Feiya Biology, FY3320-A), Rat 5HT ELISA Kit (Jiangsu Feiya Biology, FY3318-A), Rat COX-2 ELISA Kit (Jiangsu Feiya Biology, FY8509-A), Rat PGE2 ELISA Kit (Jiangsu Feiya Biology, FY2944-A), hematoxylin-eosin (HE) staining kit (Absin, abs9217), Masson trichrome staining solution (absin, abs9347).

\section{Establishment of Rat MTrPs Model}

Model establishment was conducted with the method of blunt striking and eccentric exercise modified and verified by Huang Qiangmin's team. ${ }^{19}$ The specific operations are as follows: (1) Adaptation period (1 week): Rats were given adaptive feeding to reduce the stress response to the environment, and then placed on a treadmill set (a slope of $0^{\circ}$ ) to start to train with a speed of $16 \mathrm{~m} / \mathrm{min}, 15 \mathrm{~min}$ each time, once in two days, three times in total. (2) Modeling period (8 weeks): Blunt strikes were performed on day 1 of each week after the rats anesthetized with isoflurane (induction with $4 \%$ isoflurane) and immobilized at the base of the striking apparatus, and the vastus medialis muscle was palpated and marked by an experienced physician. The blunt weight-bearing bar of the striking apparatus (total mass of $1200 \mathrm{~g}$ ) was allowed to fall freely from a height of $20 \mathrm{~cm}$ to strike the marked location with a kinetic energy of $2.352 \mathrm{~J}$. Eccentric exercise was performed on day 2 after striking, with the treadmill set at a slope of $-16^{\circ}$ and a speed of $16 \mathrm{~m} / \mathrm{min}$ for $90 \mathrm{~min}$. The rats were driven with mechanical stimuli and sound during the training to ensure the quality and quantity. For the remaining 5 days, normal feeding was provided. (3) Recovery period (4 weeks): The rats were fed normal diet every day. (4) Model evaluation: At the end of the recovery period, on palpation of the marked area by the aforementioned physician, the presence of a distinct taut band or bulging nodule, and the presence of spontaneous potentials (endplate noise or spikes) on electromyographic testing were indicative of successful modelling of rats with MTrPs.

\section{Intervention}

Rats in the blank and MTrPs model groups were not intervened while the rats in the press group were given local pressing on MTrPs as below. The rats were anesthetized with isoflurane (induction with $4 \%$ isoflurane and maintenance with $2 \%$ isoflurane) and then fixed in the supine position on a plank. The operative site (marked) was locally cleaned and shaved to expose the taut band or the area of nodules (the area near the middle of the muscle is preferred). A self-made pressing device was used for the pressing intervention according to the previous study. ${ }^{20}$ The pressing parameters were set as follows according to those set by the clinical massage practitioner: pressing head diameter $0.8 \mathrm{~cm}$, pressing force $0.7 \mathrm{~kg}$, pressing angle $90^{\circ}$ (perpendicular to the skin), 10 times $/ \mathrm{min}$ in frequency; consecutive operations, pressing intervention time $7.5 \mathrm{~min} /$ time, 1 time $/ 2 \mathrm{~d}, 7$ times in total. The pressing intervention was supervised by the practitioner to prevent accidental events.

\section{Index Assessment}

Pressure Pain Threshold (PPT)

The measurement of PPT was completed by two experimenters. Briefly, the rats were fixed to keep at a supine position by one experimenter, and then were held in a quiet state with the head covered by a sleeve and the hindquarters exposed. The muscle taut band or contracted nodule at the modeling site was marked by palpation by the other experimenter, and then pressed with the mechanical pressure pain threshold meter (Tianjin Mingtong Century Technology Co., Ltd, YT-10C). Corresponding site was selected in rats of the blank group as control. As the pressure increased, values were recorded when rat lifted and pulled back of the paw or vocalized. ${ }^{21}$ Each rat was tested three times with an interval of $10 \mathrm{~min}$. The average PPT was calculated.

\section{Soft Tissue Tension $\mathrm{D}_{0.2}$}

After the soft tissue tension meter (Tianjin Mingtong Century Technology Co., Ltd, JZL-III) was connected, debugged and calibrated, the rats were fixed with a rat cuff. The hair of the left hind limb was shaved to find the taut band or contracted nodule. The detection area was pressed vertically, taking $3 \mathrm{~s}$ both for applying and removing the pressure. The force-displacement curve was automatically generated. According to the instrument instructions and our previous research results, the displacement $\mathrm{D}_{0.2}$ corresponding to $0.2 \mathrm{~kg}$ in the curve was taken as the observation point to better reflect the tension of rat muscle and soft tissue. Each rat was tested three times with an interval of $10 \mathrm{~min}$. The average $\mathrm{D}_{0.2}$ was calculated. 


\section{Electromyogram (EMG)}

Electromyography (BIOPAC, MP150) was applied. The rats were anesthetized with isoflurane (induction with $4 \%$ isoflurane and maintenance with $2 \%$ isoflurane) and fixed on a plank, followed by hair removal of the left hind limb. The muscle taut band or contracted nodule was determined by palpation. The tail was fixed with the earth wire, and the electrode was inserted into the taut band. If there is a local convulsive response, the inserted site was marked and the electrode was retained, with another electrode inserted at $3-5 \mathrm{~mm}$ away from the side as the reference. Resting-state EMG signal was recorded for $2 \mathrm{~min}$. If there is no obvious muscle taut zone or contracted nodule by palpation in the blank group, the spontaneous potential corresponding to the markers of other groups was recorded.

\section{Tissue Sample Collection}

Once the final EMG test was finished, rats were executed by air embolization, and the tissue samples were collected from the left vastus medialis muscle taut band or area of nodules marked. The samples were cut into approximately $1 \mathrm{~cm}^{3}$ in a cryogenic environment and placed in prechilled saline, washed three times and dried with filter paper. The samples were then sub-packed and placed in $4 \%$ paraformaldehyde or stored in a $-80^{\circ} \mathrm{C}$ refrigerator.

\section{$\mathrm{HE}$ and Masson Staining}

HE staining: Tissue samples taken out from $4 \%$ paraformaldehyde were processed by following steps: paraffinembedded, sliced, dewaxed to water by conventional dehydration and embedding; stained in aqueous hematoxylin solution for $5 \mathrm{~min}$, rinsed in running water; divided in hydrochloric acid ethanol for $3 \mathrm{~s}$, rinsed in running water for $15 \mathrm{~min}$; immersed in distilled water for $5 \mathrm{~min}$; dehydrated in $70 \%$ and $90 \%$ alcohol for 10 min, respectively; stained in alcohol eosin staining solution for 2 min; dehydrated with anhydrous alcohol, processed for transparency by xylene and sealed with neutral gum.

Masson staining: Tissue samples taken out from 4\% paraformaldehyde were processed by following steps: paraffin-embedded, sliced, dewaxed to water by conventional dehydration and embedding; stained with Weigert iron hematoxylin (Weigert iron hematoxylin A and B solution mixed in equal proportions) for $5 \mathrm{~min}$, washed with running water; treated with $1 \%$ hydrochloric alcohol, rinsed with running water for a few minutes; stained with $\mathrm{Li}$ Chunhong Acid Fuchsin Staining Solution for $5 \mathrm{~min}$, rinsed with running water; treated with phosphomolybdic acid solution for about $5 \mathrm{~min}$; counterstained with aniline blue solution for $5 \mathrm{~min}$, treated with $1 \%$ glacial acetic acid for $1 \mathrm{~min}$; dehydrated with $95 \%$ alcohol for several times, followed by dehydration with anhydrous alcohol, transparency by xylene and sealing with neutral gum. Each slice was observed by a light microscope and three random fields were selected for analysis. The ratio of myofibril area to collagen fiber area was calculated by Image-Pro Plus 6.0 software.

Western Blotting for Collagen I, Collagen III, $\alpha$-SMA, $\mathrm{FN}$ and MHC Test in MTrPs Tissue

The tissue samples were taken out from the $-80^{\circ} \mathrm{C}$ refrigerator, fully cut it into small pieces, and then lysed. After centrifugation, BCA kit was used for protein quantification. According to the quantitative results, the protein samples were boiled for $10 \mathrm{~min}$ with an appropriate amount of buffer. The supernatant was electrophoresed, and the proteins separated were transferred onto a biochemical membrane. After blocked with 5\% skimmed milk at $4{ }^{\circ} \mathrm{C}$ overnight, the membrane was incubated with primary antibodies at room temperature for $1 \mathrm{~h}$. Then, the HRP-labeled secondary antibody was added at room temperature for $1 \mathrm{~h}$. PBST was applied to wash the membrane. Subsequently, protein bands were developed and analyzed by Image Pro Plus 6.0 software for band gray value. The protein expression relative to GAPDH was determined according to the ratio of the band gray value of tested proteins to that of reference protein GAPDH.

\section{ELISA Kit for COX-2, PGE2, SP and 5-HT in MTrPs Tissue}

All of the experimental operations were conducted under the manufacturer's instructions. Tissue samples taken out from the $-80^{\circ} \mathrm{C}$ refrigerator were prepared into tissue homogenate. The samples were allowed to equilibrate at room temperature, and then loaded to the testing plate with standard and sample wells. Reagents were added according to standard steps and the samples were incubated in a $37^{\circ} \mathrm{C}$ water bath. Optical density value of each well was measured to obtain a standard linear regression curve and the concentration of each sample was calculated accordingly.

\section{Statistical Analysis}

SPSS 21.0 for Windows software was used for all data statistical analyses. For data conforming to a normal distribution, paired $t$ test was used for comparison between 
two groups while one-way ANOVA was adopted when there were more than two groups. In the meantime, for multiple comparisons, LSD or SNK-q method was applied when the variance was homogeneous, otherwise, Dunnett T3 method was used. For data that did not fit a normal distribution, rank sum test and Kruskal-Wallis test were respectively applied in comparisons between two groups or among multiple groups. Multiple comparisons were also performed in multiple groups. The difference was viewed statistically significant when $P<0.05$.

\section{Results}

\section{General Conditions}

Thirty SD rats were randomly divided into blank group, model group and press group, with 10 rats in each group. Following 8 weeks of blunt striking plus eccentric exercise and 4 weeks of recovery, all of the rats in model and press groups were evaluated and eligible, without deaths or accidents such as skin contusions, infections, hemorrhages or fractures.

\section{Mechanical PPT and Soft Tissue Tension $\mathrm{D}_{0.2}$}

The test results of mechanical PPT are shown in Figure 1. Before-intervention: the PPT of the model and the press groups decreased as compared to that of the blank group ( $\mathrm{P}$ $<0.05)$, while no significant difference was observed between the model and the press groups $(\mathrm{P}>0.05)$. Post-intervention: compared with before-intervention, the PPT in the press group increased $(\mathrm{P}<0.05)$ but showed no significant difference in the model group $(\mathrm{P}>0.05)$; there was a significant difference between the model and the press groups $(\mathrm{P}$ $<0.05)$. Comparison of PPT difference before and post intervention: the difference of PPT before and post intervention in the press group was higher than that in the blank and model groups $(\mathrm{P}<0.05)$. There was no significant difference in PPT difference between the blank and the model groups $(\mathrm{P}>$ 0.05). These results suggest that pressing intervention can improve the mechanical PPT of MTrPs in rats.

The soft tissue tension test results are shown in Figure 2. Before-intervention: compared with the blank group, $\mathrm{D}_{0.2}$ in the model group and the press group decreased $(\mathrm{P}<0.05)$ without significant difference between the two groups $(\mathrm{P}>$ 0.05). Post-intervention: compared with before-intervention, the $\mathrm{D}_{0.2}$ in the press group increased $(\mathrm{P}<0.05)$ but showed no difference in the model group $(\mathrm{P}>0.05)$; there was a significant difference between the model and the press groups $(\mathrm{P}$ $<0.05)$. Comparison of $\mathrm{D}_{0.2}$ difference before and post intervention: the difference of $\mathrm{D}_{0.2}$ before and post intervention in the press group was higher than that in the blank and model groups $(\mathrm{P}<0.05)$. There was no significant difference in $\mathrm{D}_{0.2}$ difference between the blank group and the model group $(\mathrm{P}>$ 0.05). These results suggest that pressing intervention can reduce the soft tissue tension in MTrPs of rats.

\section{Electromyography}

The EMG test results after intervention are shown in Figure 3. It could be seen that only the environmental background noise appeared in the blank group. The frequency and amplitude of spontaneous potential in the model and the press groups increased compared with the

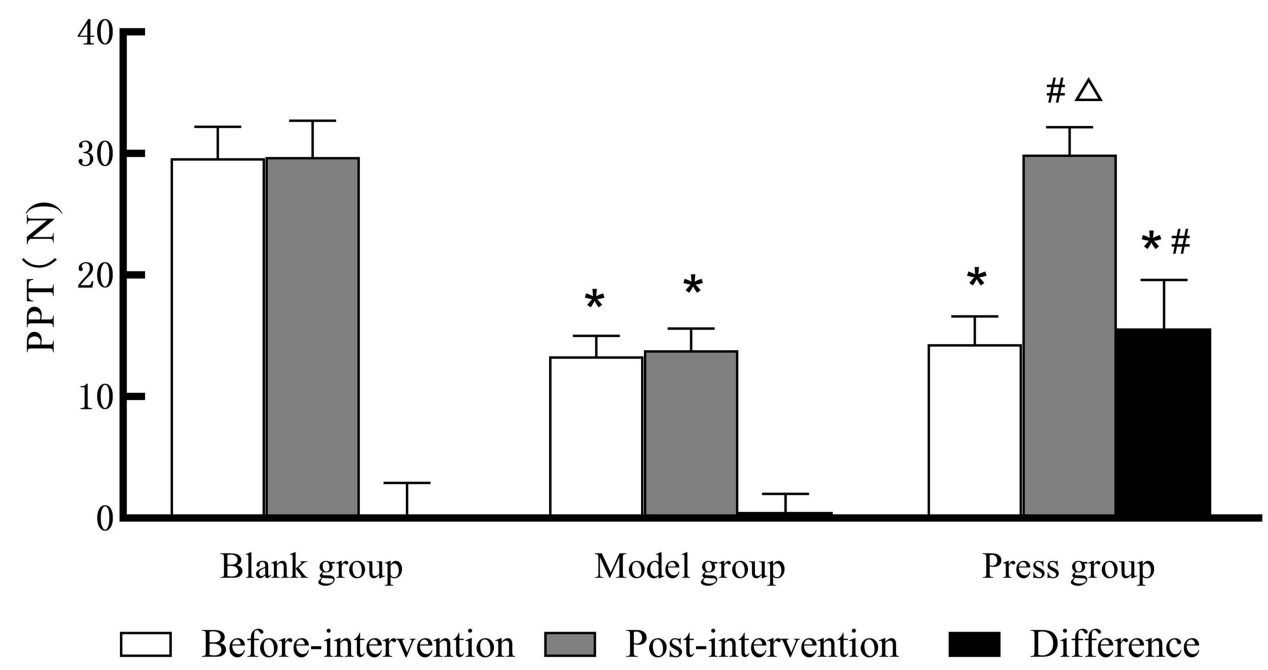

Figure I PPT in each group (blank, model and press; $\mathrm{n}=10$ per group) determined by the mechanical tenderness threshold tester. $* P<0.05$ versus blank group; ${ }^{*} P<0.05$ versus model group; ${ }^{\Delta} P<0.05$ versus before-intervention. 


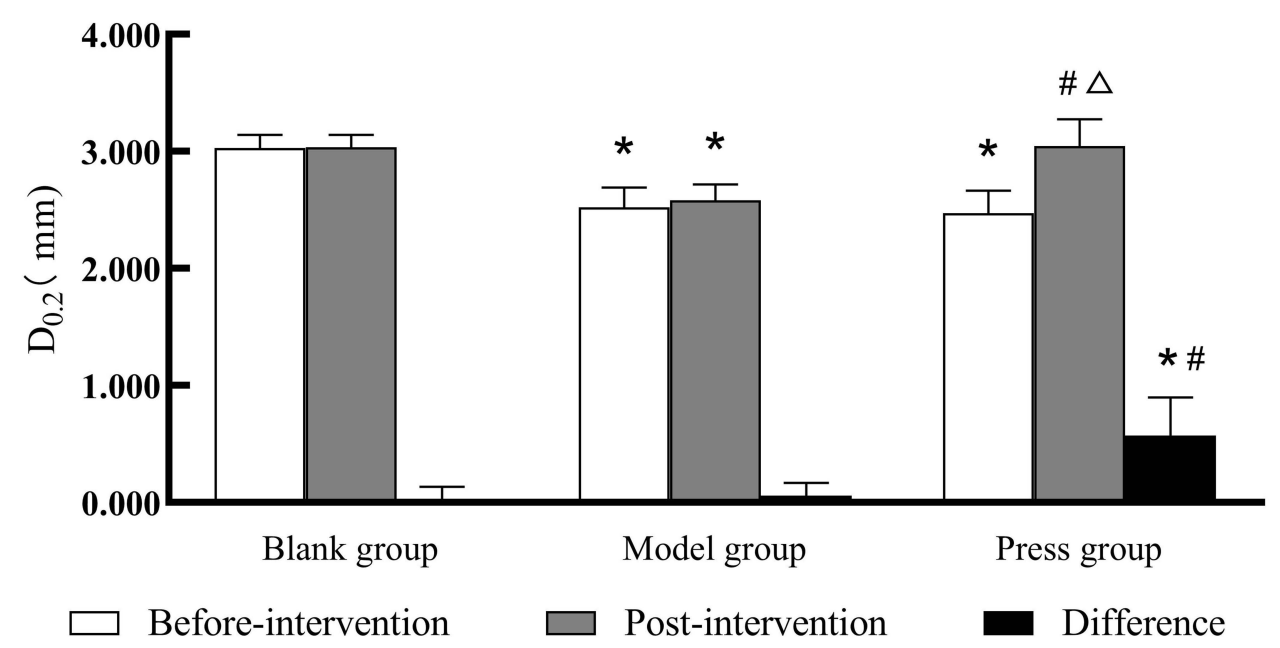

Figure $2 D_{0.2}$ in each group (blank, model and press; $\mathrm{n}=10$ per group) determined by the soft tissue tension tester. ${ }^{* P}<0.05$ versus blank group; ${ }^{\#} P<0.05$ versus model group; ${ }^{\Delta} P<0.05$ versus before-intervention.

A

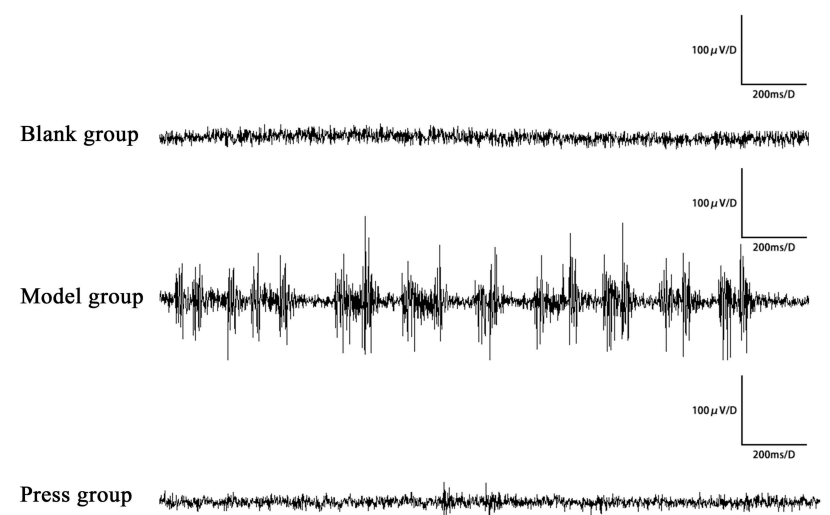

B
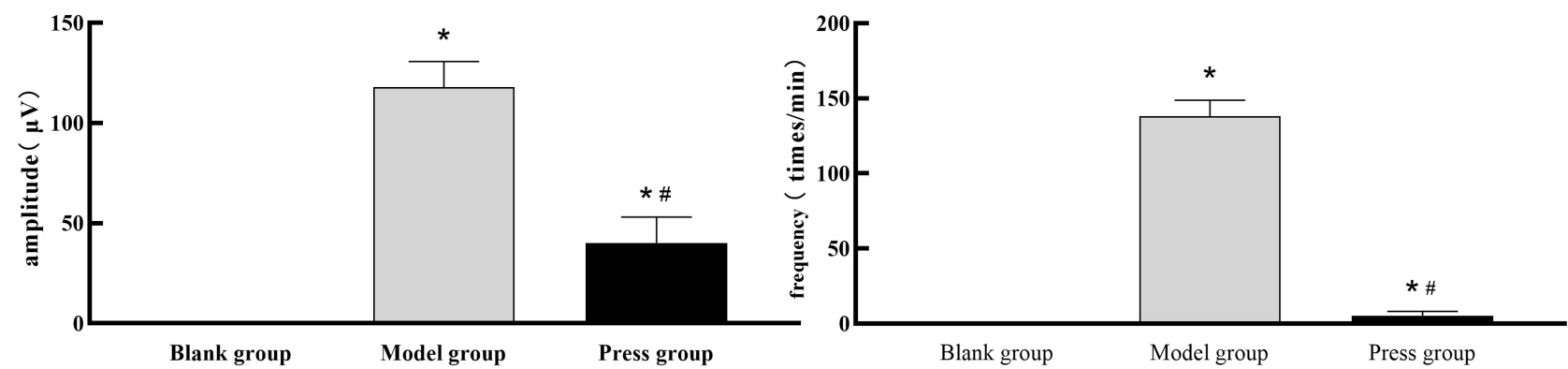

$\square$ Blank group $\square$ Model group

Press group

Figure 3 Electromyography recordings of the three groups (blank, model and press; $n=10$ per group) determined by the MPI 50 data acquisition systems. (A) Environmental background noise appeared in the blank group. Spontaneous electrical activity was detected in the model group and press group.(B) Frequency and amplitude of spontaneous potential in each group. $* P<0.05$ versus blank group; ${ }^{\#} P<0.05$ versus model group.

blank group ( $\mathrm{P}<0.05)$, while those were increased much significantly in the press group $(\mathrm{P}<0.05)$, suggesting that pressing intervention can reduce the amplitude and frequency of spontaneous potential in MTrPs of rats.

\section{$\mathrm{HE}$ and Masson Staining}

The results are shown in Figure 4. HE staining: The nuclei were presented in purple blue, and the cytoplasm and extracellular matrix were in red. The myocytes in the 
A
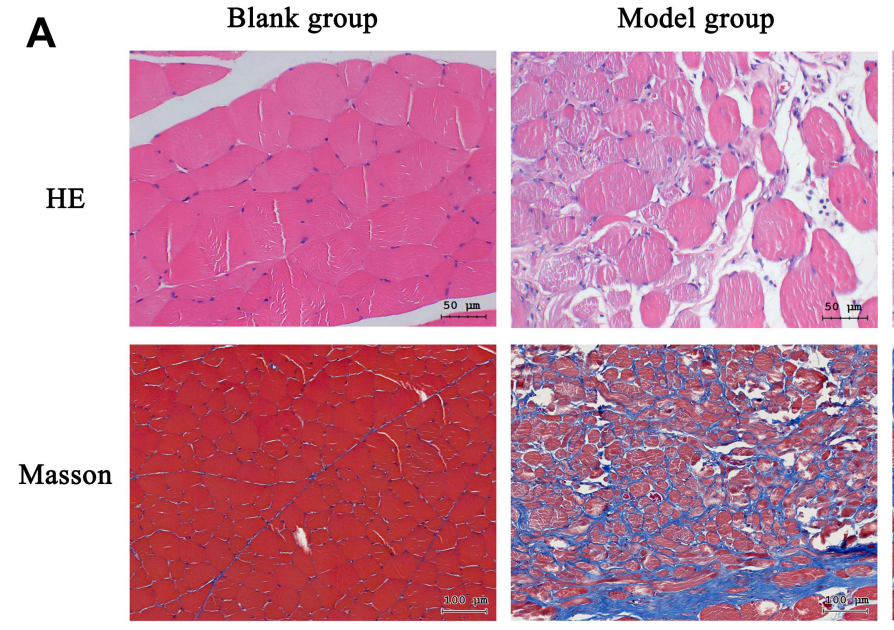

B

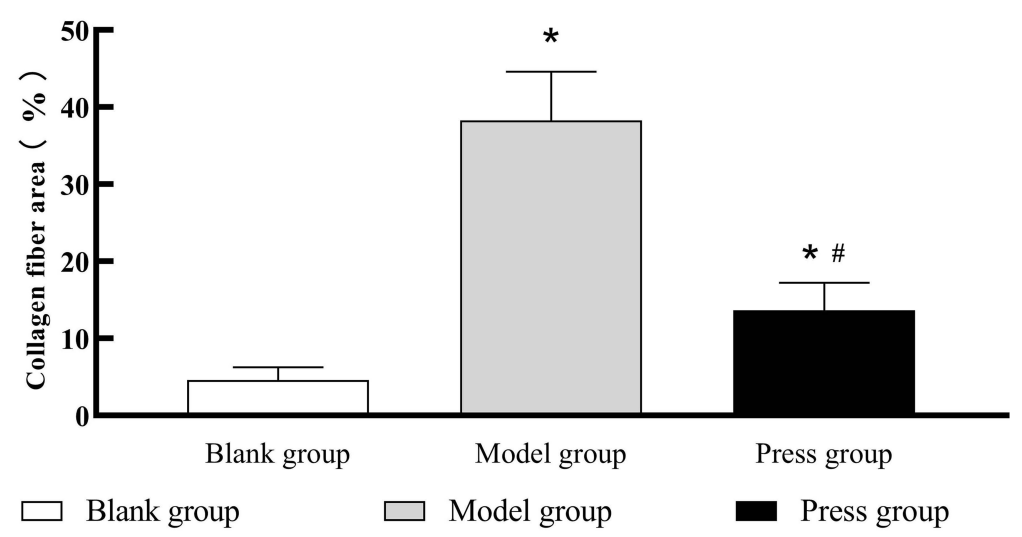

Figure 4 Microscopy images of cross-sections of muscle fibers with HE and Masson staining. (A) In the model group, enlarged, round, deeply stained myocytes could be observed with small irregular ones around. The thick myointima and nuclear migration appeared with inflammatory cells, interstitial proliferation and large-area adhesions. The integrity and continuity of skeletal muscle fibers were destroyed along with proliferated collagen fibers leading to widened cellular gap and a collagen fiber deposition area presented in blue. In the press group, the cell morphology tended to be normal and the inflammatory cells disappeared while the blue area was reduced. (B) Collagen fiber area (collagen fiber area/ total area) in each group (blank, model and press; $n=6$ per group). ${ }^{*} P<0.05$ versus blank group; ${ }^{\#} P<0.05$ versus model group.

normal tissue displayed a regular polygonal morphology arranged in order and unanimous size with multiple nuclei located under the muscle membrane. In the model group, enlarged, round, deeply stained myocytes could be observed with small irregular ones around. The thick myointima and nuclear migration appeared with inflammatory cells, interstitial proliferation and large-area adhesions. However, the cell morphology tended to be normal and the inflammatory cells disappeared in the press group. Masson staining: The normal nuclei were in blue while the skeletal muscle fibers and collagen fibers were in red and blue respectively. In the blank group, the nuclei were evenly distributed with a clear boundary, the muscle fibers were in red and orderly arranged but the collagen fibers were rare. In the model group, the integrity and continuity of skeletal muscle fibers were destroyed along with proliferated collagen fibers leading to widened cellular gap and a collagen fiber deposition area presented in blue. The area of collagen fibers was higher than that of the normal group $(\mathrm{P}<0.05)$. The muscle fibers in the press group were orderly arranged with a uniform thickness and tended to be normally structured, with the fibrosis area less than that in the model group $(\mathrm{P}<0.05)$. These results suggest that pressing intervention can alleviate the chronic inflammation and fibrosis in MTrPs of rats.

\section{The Expression of Collagen I, Collagen III, FN, $\alpha-S M A$ and MHC}

Compared with the blank group, the expression of collagen I, collagen III, FN and $\alpha$ - SMA increased $(\mathrm{P}<0.05)$ while the expression of $\mathrm{MHC}$ decreased in the model group $(\mathrm{P}<0.05)$. However, the press group showed 


\section{Blank Model Press}
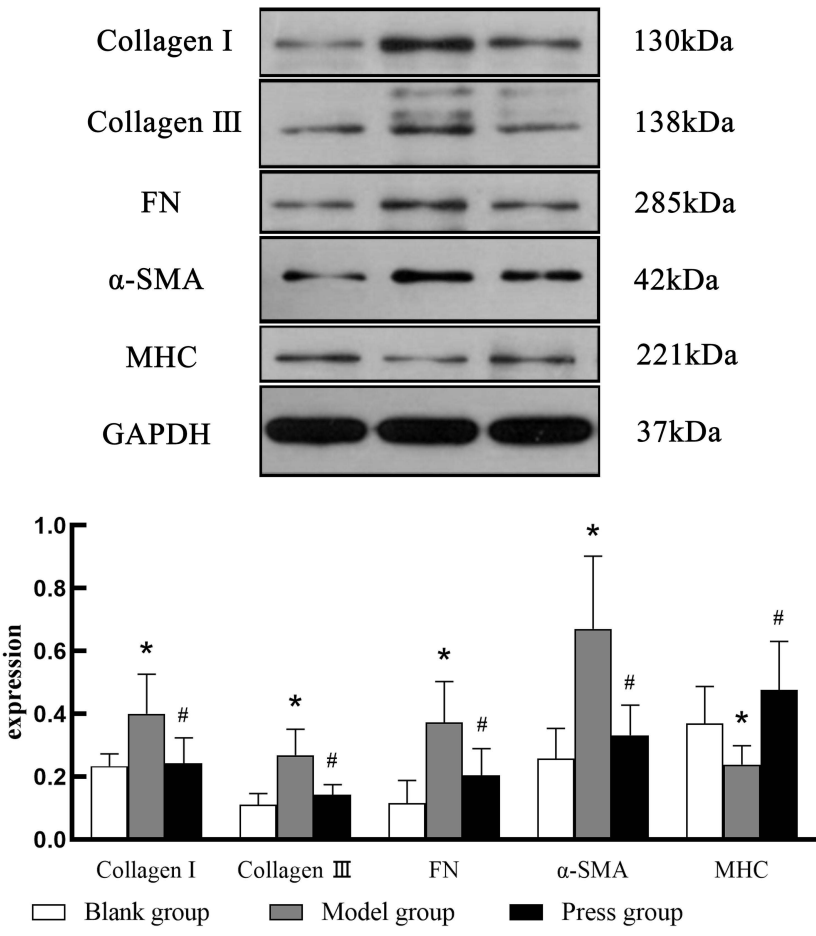

Figure 5 Collagen I, Collagen III, FN, $\alpha$-SMA and MHC expression in each group (blank, model and press; $\mathrm{n}=8$ per group). ${ }^{*} P<0.05$ versus blank group; ${ }^{\#} P<0.05$ versus model group.

decreased expression of collagen I, collagen III, FN and $\alpha$ SMA $(\mathrm{P}<0.05)$ while increased expression of MHC $(\mathrm{P}<$ 0.05) as compared to the model group (Figure 5). Altogether, these results illustrate that pressing intervention can reduce the soft tissue tension and extracellular matrix content to enhance the repair ability of skeletal muscle in MTrPs of rats.
The Content of COX-2, PGE2, SP and 5-HT

Compared with the blank group, the content of COX-2, PGE2, SP and 5-HT in the model group increased in both the model $(\mathrm{P}<0.05)$ and the press groups, while the SP and 5-HT increase in the press group was not significant $(\mathrm{P}>0.05)$. Between the model and the press groups, the content of all four substances decreased significantly in the press group $(\mathrm{P}<0.05)$ (Figure 6). To sum up, pressing intervention can reduce the content of multiple inflammatory factors in MTrPs of rats.

\section{Discussion}

Massage therapy has been widely used for internal, external, gynecological and pediatric diseases in various regions and countries all over the world. Pressing-based techniques such as Chinese medicine pressing, Thai fingerpressing and ischemic pressing are common in treatment of various types of pain. ${ }^{22}$ There is evidence that pressingbased techniques are safe and effective in relieving symptoms caused by MTrPs. ${ }^{23}$ The pressing-based deactivation of MTrPs has now been a hot research spot in the field of massage while it is still hard to clarify due to study method. Generally, pressing on MTrPs suppresses pain at two levels: peripheral and central. At the peripheral level, analgesia by pressing is commonly accompanied by increased local blood flow, improved metabolic environment, and enhanced local metabolism. ${ }^{24,25}$ In addition, local sympathetic remodeling happens in MTrPs, which will cause sympathetic hyperinnervation and excessive release of local acetylcholine, finally leading to a variety of relevant symptoms. ${ }^{26}$ Additionally, pressing on MTrPs can enhance the excitability of parasympathetic neurons
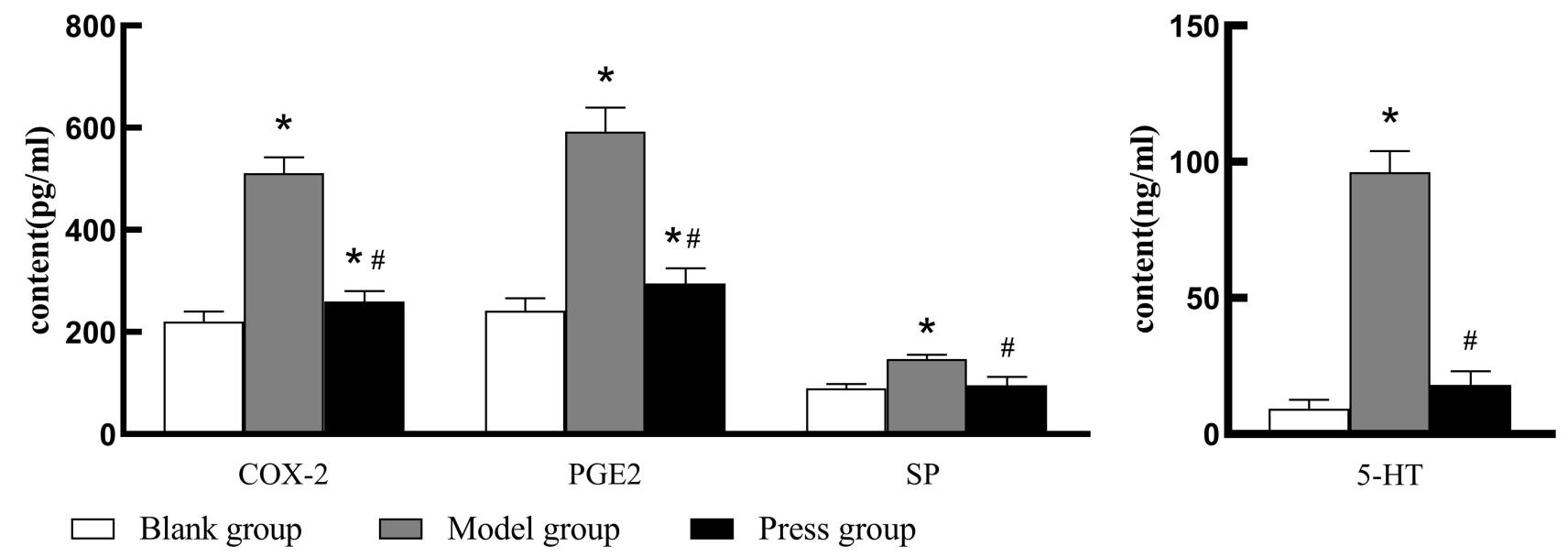

Figure 6 COX-2, PGE2, SP and 5-HT content in each group (blank, model and press; $\mathrm{n}=8$ per group). ${ }^{*} \mathrm{P}<0.05$ versus blank group; ${ }^{\#} P<0.05$ versus model group. 
while inhibiting the excitability of sympathetic nerves, resulting in decrease of acetylcholine release at the motor endplates, ultimately improving the symptoms. ${ }^{27}$ At the central level, current research believed that pressing on MTrPs decreases subjective pain by affecting autonomic nerves system activities via the prefrontal cortex. ${ }^{28,29}$ Certain advances have been achieved regarding the mechanism of pressing on MTrPs, but limitations remain exist. In this context, this study focused on the mechanism at the peripheral level.

Predisposing factors of MTrPs mainly are various acute and chronic injuries (eg, trauma, work-related repetitive muscle injury, eccentric exercise, physical or psychological disorders, and iatrogenic injuries). In basic animal research, neostigmine injection and blunt striking plus eccentric exercise are the two main strategies used for establishing animal models of MTrPs. ${ }^{30}$ The latter is the most popular way at present, as it conforms to the acute and chronic injury etiology of MTrPs, and the model correspondingly established shows more consistent histomorphological and electromyographic manifestations of contracture nodules and intermittent spontaneous electrical activity in MTrPs. ${ }^{19,31}$ In this context, this study established rat models of MTrPs with this method. According to a Delphi survey in 2018, ${ }^{32}$ it was concluded that at least two of the three diagnostic criteria of "a taut band", "a hypersensitive spot" and "referred pain" should be met combined with original pain of the patient in the diagnosis of MTrPs. Besides, convulsive reaction and autonomic symptoms could be used as secondary supporting evidence. In the present study, PPT and $\mathrm{D}_{0.2}$ were lower in the model group than that of the blank group with frequent spontaneous potentials. After given pressing intervention, PPT and $\mathrm{D}_{0.2}$ were elevated and spontaneous potentials frequency and amplitude were reduced, suggesting that the local soft tissue tension could be reduced by pressing intervention.

According to the integrated hypothesis, the development of MTrPs is associated with a vicious cycle involving abnormal motor endplate (neuromuscular junction), acetylcholine accumulation, impaired local circulation and energy metabolism, as well as abnormal release of vasoactive components and inflammatory factors. ${ }^{33}$ Following the formation of MTrPs, the abnormal structure and function of motor endplate will produce spontaneous potentials, $^{34}$ and the local acetylcholine accumulation will promote the continuous increase of calcium ions in muscle cells, while will cause contraction of sarcomeres leading to formation of contracture nodules, compression of blood vessels, local hypoxia, microcirculation and energy metabolism disorders. In addition, a large amount of inflammatory pain-causing substances will be released to stimulate nerve endings leading to the generation of pain. Hence, inflammatory substances are regarded as important players in the occurrence and development of MTrPs. In case of muscle injury, IL-6 and TNF- $\alpha$ will be up-regulated to concomitantly induce expression of COX2 , which promotes the production of PGE2 by monocytes and macrophages leading to recruitment of SP derived from fine fiber endings into the body fluid to stimulate mast cells and platelets, followed by release of histamine and 5-HT contributing to multiple complex networks of inflammatory factors. All networks together sensitize peripheral pain receptors to form hyperalgesia. ${ }^{35,36}$ Our experimental data showed that the COX-2, PGE2, SP, and 5-HT levels increased in model group and decreased after pressing intervention. HE staining showed a great deal of inflammatory cells in the model group, indicating that there was a significant inflammatory response in the MTrPs tissue, while pressing intervention could reduce the expression of inflammatory factors significantly. In the press group, there still existed a small number of spontaneous potentials. Additionally, HE staining revealed that the muscular cells failed to restore to normal, and the levels of COX-2 and PGE2 were higher than the control. There might be two reasons: 1) PGE2 at a certain level may promote skeletal muscle repair. It is reported that intramuscular injection of PGE2 contributed to significantly enhanced and accelerated repair of injured muscle. ${ }^{37}$ COX-2 is an upstream molecule of PGE2 and responsible for regulating myogenic signaling. ${ }^{38}$ 2) The pressing intervention in this study failed to recover partial inflammatory factors and injured muscle to normal, requiring further research on the dose-effect relationship of pressing.

Studies have shown that chronic inflammatory cell infiltration promotes fibroblast proliferation, resulting in extracellular matrix (ECM) deposition and tissue fibrosis, which hinders tissue repair process. ${ }^{39,40}$ Collagen I, collagen III and FN are important components of the ECM of skeletal muscle. While in skeletal muscle tissue, other than collagen I and collagen III, there still exists collagen IV and collagen V. Among the collagens, collagen I and collagen III are the most abundant and distributed on the endomysium and myo-fascicular membrane. They are mainly devoted to link and support myocytes and 
myofascial tissue, which is closely related to muscle biomechanical properties. ${ }^{41} \mathrm{FN}$ is another fiber-forming glycoprotein in the ECM that interacts with cell surface receptors such as integrin, collagen and fibrin, and acts as a bridge in matrix-tissue and cell-matrix interactions, such as fiber network forming. ${ }^{42}$ Myosin, composed of heavy chain (MHC) and light chain, is the most abundant contractile structure in muscle cells and the basic constituent protein of myofibril thick filaments. Changes in $\mathrm{MHC}$ are closely related to the process of muscle regeneration rendering it as the muscle regeneration marker. ${ }^{43,44}$ Once skeletal muscle is damaged, inflammatory cells infiltrate to clear necrotic tissue, inducing muscle satellite cell proliferation and differentiation into myoblasts to repair damaged muscle fibers. Fibroblasts are the major cells to produce collagen and can form a fiber network with FN. The damage at both ends of the attached fibers improves the tensile strength and plays a positive role in the early healing process of injured skeletal muscle. ${ }^{45}$ Simultaneously, $\alpha$-SMA, expressed by myofibroblasts, can also play as the specific marker of myofibroblast proliferation. ${ }^{46}$ When the acute injury gradually becomes chronic, inflammatory cells continue to infiltrate and secrete inflammatory cytokines, resulting in abnormally increased TGF- $\beta$. Thus, a large number of myofibroblasts will proliferate in damaged muscle tissue producing excessive ECM, including collagen and FN. The overexpressed FN further promotes matrix deposition, leading to muscle fibrosis and inhibiting the regeneration of injured skeletal muscle. ${ }^{47}$ Furthermore, muscle satellite cells differentiate into myofibroblasts and further promote fibrosis. ${ }^{48}$

In our present study, $\mathrm{HE}$ staining showed that there was interstitial proliferation and a large area of adhesions in the model group, while Masson staining confirmed MTrPs muscle fibrosis, which could be reduced by pressing intervention. Western blotting showed that collagen I, collagen III, FN and $\alpha$-SMA expression increased and MHC expression decreased in the model group, suggesting the proliferation of myofibroblasts, deposition of ECM and inhibition of skeletal muscle repair. This confirms the previous experimental results of other research teams from different perspectives: in the presence of MTrPs, there existed inflammatory cell infiltration, tissue fibrosis associated TGF- $\beta 1 /$ Smad pathway up-regulation, and muscle satellite cell activation while without myoblast differentiation. ${ }^{49,50}$ Additionally, we also found that collagen I, collagen III, FN and $\alpha$ - SMA expression decreased while MHC increased by pressing intervention, indicating that pressing intervention on MTrPs could inhibit myofibroblasts proliferation, reduce ECM deposition and promote skeletal muscle regeneration.

In conclusion, pressing intervention on MTrPs can alleviate chronic inflammation, inhibit fibrosis and promote skeletal muscle repair. However, our study is preliminary an in vivo animal experiment that lacks the research into time gradient and dose-effect relationship. In the future, further experiments need to be carried out to comprehensively and systematically explore the specific mechanism of pressing.

\section{Acknowledgments}

This work was supported by National Natural Science Foundation of China (grant number 81973975) and Scientific Research Project of Hunan Provincial Department of Education (grant number 20B439). The first author would like to thank his junior fellow apprentices for experiment support and researcher Li-ping for excellent technical support.

\section{Disclosure}

Dr Xiaowei Liu reports grants from National Natural Science Foundation of China, during the conduct of the study; grants from National Natural Science Foundation of China, outside the submitted work. Dr $\mathrm{Wu} \mathrm{Li}$ reports grants from National Natural Science Foundation of China, during the conduct of the study; grants from National Natural Science Foundation of China, outside the submitted work. Dr Jiangshan Li reports grants from National Natural Science Foundation of China during the conduct of the study; grants from National Natural Science Foundation of China outside the submitted work. The authors report no conflicts of interest in this work.

\section{References}

1. Claire D, Amber D. The Trigger Point Therapy Workbook. Beijing, China: Beijing Science and Technology Publishing Co.,Ltd; 2018

2. Treede RD, Rief W, Barke A, et al. Chronic pain as a symptom or a disease: the IASP classification of chronic pain for the International Classification of Diseases (ICD-11). Pain. 2019;160(1):19-27. doi:10. 1097/j.pain.0000000000001384

3. Do TP, Heldarskard GF, Kolding LT, et al. Myofascial trigger points in migraine and tension-type headache. J Headache Pain. 2018;19(1):84. doi:10.1186/s10194-018-0913-8

4. Ribeiro DC, Belgrave A, Naden A, et al. The prevalence of myofascial trigger points in neck and shoulder-related disorders: a systematic review of the literature. BMC Musculoskelet Disord. 2018;19(1):252. doi:10.1186/s12891-018-2157-9

5. Badil Güloğlu S, Tunç S. The assessment of affective temperament and life quality in myofascial pain syndrome patients. Int $J$ Psychiatry Clin Pract. 2020;1-6. doi:10.1080/13651501.2020.1833039 
6. Zhang Y, Du NY, Chen C, et al. Acupotomy alleviates energy crisis at rat myofascial trigger points. Evid Based Complement Alternat Med. 2020;6:5129562. doi:10.1155/2020/5129562.

7. Itoh K, Okada K, Kawakita K. A proposed experimental model of myofascial trigger points in human muscle after slow eccentric exercise. Acupunct Med. 2004;22(1):2-12; discussion 12-3. doi:10.1136/ aim.22.1.2

8. Bron C, Dommerholt JD. Etiology of myofascial trigger points. Curr Pain Headache Rep. 2012;16(5):439-444. doi:10.1007/s11916-012-0289-4

9. Mahdy M. Skeletal muscle fibrosis: an overview. Cell Tissue Res. 2019;375(3):575-588. doi:10.1007/s00441-018-2955-2

10. Duarte F, West D, Linde LD, Hassan S, Kumbhare DA. Re-examining myofascial pain syndrome: toward biomarker development and mechanism-based diagnostic criteria. Curr Rheumatol Rep. 2021;23 (8):69. doi:10.1007/s11926-021-01024-8

11. Cao QW, Peng BG, Wang L, et al. Expert consensus on the diagnosis and treatment of myofascial pain syndrome. World J Clin Cases. 2021;9(9):2077-2089. doi:10.12998/wjcc.v9.i9.2077

12. Nasb M, Qun X, Ruckmal Withanage C, et al. Dry cupping, ischemic compression, or their combination for the treatment of trigger points: a pilot randomized trial. J Altern Complement Med. 2020;26(1):4450. doi:10.1089/acm.2019.0231

13. Esparza D, Aladro-Gonzalvo AR, Rybarczyk Y. Effects of local ischemic compression on upper limb latent myofascial trigger points: a study of subjective pain and linear motor performance. Rehabil Res Pract. 2019;2019:5360924. doi:10.1155/2019/5360924.

14. da Silva AC, De Noronha M, Liberatori-Junior RM, et al. The effectiveness of ischemic compression technique on pain and function in individuals with shoulder pain: a systematic review. $J$ Manipulative Physiol Ther. 2020;43(3):234-246. doi:10.1016/j.jmpt.2019.10.013

15. Togha M, Bahrpeyma F, Jafari M, et al. A sonographic comparison of the effect of dry needling and ischemic compression on the active trigger point of the sternocleidomastoid muscle associated with cervicogenic headache: a randomized trial. J Back Musculoskelet Rehabil. 2020;33(5):749-759. doi:10.3233/BMR-171077

16. Alghadir AH, Iqbal A, Anwer S, et al. Efficacy of combination therapies on neck pain and muscle tenderness in male patients with upper trapezius active myofascial trigger points. Biomed Res Int. 2020;2020:9361405. doi:10.1155/2020/9361405

17. Martín-Pintado-Zugasti A, López-López A, González Gutiérrez JL, et al. The role of psychological factors in the perception of postneedling soreness and the influence of postneedling intervention. PMR. 2017;9(4):348-355. doi:10.1016/j.pmrj.2016.07.529

18. Jiang YT, Li TL, Li JS, et al. Effect of Pressing Manipulation in Massage on Ultrastructure of Skeletal Muscle in Rats with Chronic Myofascial Trigger Points. Journal of Hunan University of Chinese Medicine. 2021;41(01):85-90.

19. Zhang H, Lü JJ, Huang QM, Liu L, Liu QG, Eric OA. Histopathological nature of myofascial trigger points at different stages of recovery from injury in a rat model. Acupunct Med. 2017;35(6):445-451. doi:10.1136/acupmed-2016-011212

20. Quan-rui J, Jiang-shan L, Si-hong C, et al. Orthogonal test on local temperature influenced by different parameters and manipulation types of An-pressing Xinshu (BL 15). J Acupuncture Tuina Sci. 2019;17(3):147-154. doi:10.1007/s11726-019-1105-Z

21. Ishikawa K, Kajiwara Y, Sakamoto J, et al. Low-intensity muscle contraction exercise following the onset of arthritis improves hyperalgesia via reduction of joint inflammation and central sensitization in the spinal cord in a rat model. Neurosci Lett. 2019;706:18-23. doi:10.1016/j.neulet.2019.04.031

22. Ziaeifar M, Arab AM, Mosallanezhad Z, Nourbakhsh MR. Dry needling versus trigger point compression of the upper trapezius: a randomized clinical trial with two-week and three-month follow-up. J Man Manip Ther. 2019;27(3):152-161. doi:10.1080/10669817.2018.1530421
23. Benito-de-pedro M, Becerro-de-bengoa-vallejo R, Losa-Iglesias ME, et al. Effectiveness between dry needling and ischemic compression in the triceps surae latent myofascial trigger points of triathletes on pressure pain threshold and thermography: a single blinded randomized clinical trial. J Clin Med. 2019;8(10):1632. doi:10.3390/ jcm8101632

24. Moraska AF, Hickner RC, Rzasa-Lynn R, et al. Increase in lactate without change in nutritive blood flow or glucose at active trigger points following massage: a randomized clinical trial. Arch Phys Med Rehabil. 2018;99(11):2151-2159. doi:10.1016/j.apmr.2018.06.030

25. Moraska AF, Hickner RC, Kohrt WM, et al. Changes in blood flow and cellular metabolism at a myofascial trigger point with trigger point release (ischemic compression): a proof-of-principle pilot study. Arch Phys Med Rehabil. 2013;94(1):196-200. doi:10.1016/j.apmr.2012.08.216

26. Cao L, Gao Y, Wu K, et al. Sympathetic hyperinnervation in myofascial trigger points. Med Hypotheses. 2020;139:109633. doi:10.10 16/j.mehy.2020.109633

27. Takamoto K, Sakai S, Hori E, et al. Compression on trigger points in the leg muscle increases parasympathetic nervous activity based on heart rate variability. J Physiol Sci. 2009;59(3):191-197. doi:10. 1007/s12576-009-0025-y

28. Morikawa Y, Takamoto K, Nishimaru H, et al. Compression at myofascial trigger point on chronic neck pain provides pain relief through the prefrontal cortex and autonomic nervous system: a pilot study. Front Neurosci. 2017;11:186. doi:10.3389/fnins.2017.00186

29. Kodama K, Takamoto K, Nishimaru H, et al. Analgesic effects of compression at trigger points are associated with reduction of frontal polar cortical activity as well as functional connectivity between the frontal polar area and insula in patients with chronic low back pain: a randomized trial. Front Syst Neurosci. 2019;13:68. doi:10.3389/ fnsys. 2019.00068

30. Margalef R, Sisquella M, Bosque M, et al. Experimental myofascial trigger point creation in rodents. J Appl Physiol. 2019;126(1):160169. doi:10.1152/japplphysiol.00248.2018

31. Huang QM, Ye G, Zhao ZY, Lv JJ, Tang L. Myoelectrical activity and muscle morphology in a rat model of myofascial trigger points induced by blunt trauma to the vastus medialis. Acupunct Med. 2013;31(1):65-73. doi:10.1136/acupmed-2012-010129

32. Fernández-de-las-peñas $\mathrm{C}$, Dommerholt J. International consensus on diagnostic criteria and clinical considerations of myofascial trigger points: a delphi study. Pain Med. 2018;19(1):142-150. doi:10.1093/ $\mathrm{pm} / \mathrm{pnx} 207$

33. Weller JL, Comeau D, Otis J. Myofascial pain. Semin Neurol. 2018;38(6):640-643. doi:10.1055/s-0038-1673674

34. Liu QG, Huang QM, Liu L, Nguyen TT. Structural and functional abnormalities of motor endplates in rat skeletal model of myofascial trigger spots. Neurosci Lett. 2019;711:134417. doi:10.1016/j.neulet. 2019.134417

35. Tsuge K, Inazumi T, Shimamoto A, Sugimoto Y. Molecular mechanisms underlying prostaglandin E2-exacerbated inflammation and immune diseases. Int Immunol. 2019;31(9):597-606. doi:10.1093/ intimm/dxz021

36. Zieglgänsberger W. Substance $P$ and pain chronicity. Cell Tissue Res. 2019;375(1):227-241. doi:10.1007/s00441-018-2922-y

37. Ho A, Palla AR, Blake MR, et al. Prostaglandin E2 is essential for efficacious skeletal muscle stem-cell function, augmenting regeneration and strength. Proc Natl Acad Sci U S A. 2017;114(26):6675-6684. doi:10.1073/pnas.1705420114.

38. Mo C, Wang Z, Bonewald L, Brotto M. Multi-staged regulation of lipid signaling mediators during myogenesis by COX-1/2 pathways. Int J Mol Sci. 2019;20(18):4326. doi:10.3390/ijms20184326

39. Forcina L, Miano C, Scicchitano BM, Musarò A. Signals from the niche: insights into the role of IGF-1 and IL-6 in modulating skeletal muscle fibrosis. Cells. 2019;8(3):232. doi:10.3390/cells8030232 
40. Wynn TA, Ramalingam TR. Mechanisms of fibrosis: therapeutic translation for fibrotic disease. Nat Med. 2012;18(7):1028-1040. doi: $10.1038 / \mathrm{nm} .2807$

41. Gillies AR, Lieber RL. Structure and function of the skeletal muscle extracellular matrix. Muscle Nerve. 2011;44(3):318-331. doi:10.100 2/mus.22094

42. Theocharis AD, Manou D, Karamanos NK. The extracellular matrix as a multitasking player in disease. FEBS J. 2019;286(15):28302869. doi: $10.1111 /$ febs. 14818

43. Wang R, Luo D, Xiao C, et al. The time course effects of electroacupuncture on promoting skeletal muscle regeneration and inhibiting excessive fibrosis after contusion in rabbits. Evid Based Complement Alternat Med. 2013;2013:869398. doi:10.1155/2013/ 869398.

44. Schiaffino S. Muscle fiber type diversity revealed by anti-myosin heavy chain antibodies. FEBS J. 2018;285(20):3688-3694. doi:10. $1111 /$ febs. 14502

45. Hinz B. Myofibroblasts. Exp Eye Res. 2016;142:56-70. doi:10.1016/ j.exer.2015.07.009.
46. Angelini A, Trial J, Ortiz-Urbina J, et al. Mechanosensing dysregulation in the fibroblast: a hallmark of the aging heart. Ageing Res Rev. 2020;63:101150. doi:10.1016/j.arr.2020.101150

47. David C, Eric L, Jeffrey L, et al. Traumatic muscle fibrosis: from pathway to prevention. J Trauma Acute Care Surg. 2017;82(1):174. doi:10.1097/TA.0000000000001290.

48. Delaney K, Kasprzycka P, Ciemerych MA, Zimowska M. The role of TGF- $\beta 1$ during skeletal muscle regeneration. Cell Biol Int. 2017;41 (7):706-715. doi:10.1002/cbin.10725

49. Yuan SG, Yan LM, Wu K, et al. Effects of chemical sympathectomy on inflammation of myofascial trigger points and myogenic differentiation of muscle satellite cells. The Journal of Practical Medicine. 2020;36(15):2059-2065.

50. Jing YJ, Chen MX, Cao L, et al. Effect of Moxibustion on Expression of TGF- $\beta 1$ /Smad4 in Myofascial Pain Point. Chinese Archives of Traditional Chinese Medicine. 2018;36(12):3019-3022, 3110-3112.

\section{Publish your work in this journal}

The Journal of Pain Research is an international, peer reviewed, open access, online journal that welcomes laboratory and clinical findings in the fields of pain research and the prevention and management of pain. Original research, reviews, symposium reports, hypothesis formation and commentaries are all considered for publication. The manuscript management system is completely online and includes a very quick and fair peer-review system, which is all easy to use. Visit http:// www.dovepress.com/testimonials.php to read real quotes from published authors. 